\title{
Anoftalmia clínica bilateral associada à hidrocefalia congênita em cão
}

\author{
Bilateral clinical anophthalmia associated with congenital hydrocephalus in the dog
}

\author{
Mariana Isa Poci Palumbo ${ }^{\mathrm{I}}$ Jorge Piovesan Conti ${ }^{\mathrm{I}}$ Danuta Pulz Doiche ${ }^{\mathrm{I}}$ Maria Jaqueline Mamprim ${ }^{\mathrm{I}}$ \\ Maria Lúcia Gomes Lourenço ${ }^{I}$ Luiz Henrique de Araújo Machado ${ }^{I}$
}

RESUMO

A ausência completa do bulbo ocular é muito rara em cães e gatos, enquanto a hidrocefalia é comumente observada como distúrbio congênito em cães de raças miniatura ou braquicefálicas, com menos de um ano de idade. O presente trabalho relata a ocorrência de anoftalmia clínica bilateral associada à hidrocefalia congênita em um cão da raça poodle, sendo este o primeiro relato de caso da associação dessas alterações no Brasil.

Palavras-chave: neurologia, cão, oftalmologia, alterações congênitas.

\section{ABSTRACT}

The complete absence of the eyeball is rare in dogs and cats, and hydrocephalus is commonly seen as a congenital disorder in toy or brachycephalic dogs before one year old. This paper describes for the first time in Brazil the occurrence of bilateral clinical anophthalmia associated to congenital hydrocephalus in a dog.

Key words: neurology, dog, ophthalmology, congenital disorders.

A ausência completa do bulbo ocular ou anoftalmia é rara em cães e gatos (MOULD, 2002; CHRISMAN et al., 2005; DEWEY, 2006; SPIESS, 2007; COOK, 2007; FERNÁNDEZ; BERNARDINI, 2010), sendo decorrente do crescimento inadequado da vesícula ou cúpula óptica, em estágio precoce da gestação, ou por falha posterior da expansão desse elemento óptico (SPIESS, 2007). O exame histopatológico dos tecidos orbitários pode revelar traços de estruturas ópticas rudimentares (NARFSTROM et al., 2001; MOULD, 2002; SPIESS, 2007). Em todas as espécies, anormalidades oculares congênitas múltiplas podem ser observadas, sendo, com frequencia, de origem hereditária (SLATTER, 2005). Os cães afetados e seus descendentes próximos com esta afecção não devem ser utilizados para reprodução (PRIESTER, 1972; GELATT, 2003).

O termo hidrocefalia geralmente é empregado para indicar aumento de volume dos ventrículos cerebrais, em particular dos ventrículos laterais (CHRISMAN et al., 2005). A ventriculomegalia resulta da obstrução do fluxo de líquido cefalorraquidiano (LCR) no sistema ventricular (como nos casos de estenose do arqueduto mesencefálico) e/ou absorção insuficiente do LCR no sistema nervoso, nas vilosidades aracnóides. A terapia farmacológica é eficaz em alguns casos, contudo, a realização de procedimentos cirúrgicos visando à drenagem do líquido cefalorraquidiano para controle dos sinais clínicos, muitas vezes, é indicada (FILGUEIRAS et al., 2009). O prognóstico da hidrocefalia congênita em cães e gatos é variável, sendo, contudo, geralmente considerado reservado (DEWEY, 2006).

Foi atendido pelo Serviço de Clínica Médica de Pequenos Animais da Faculdade de Medicina Veterinária e Zootecnia da UNESP, campus de

IFaculdade de Medicina Veterinária e Zootecnia (FMVZ), Universidade Estadual Paulista “Júlio de Mesquita Filho” (UNESP), 18618-970, Botucatu, SP, Brasil. E-mail: palumboma11@yahoo.com.br. *Autor para correspondência. 
Botucatu, um cão da raça poodle com três meses de idade com histórico de incoordenação motora e fraqueza. O cão havia nascido com peso corpóreo inferior quando comparado a outros filhotes da ninhada, sem anormalidades clínicas.

Durante o exame físico, observou-se fontanela presente e pálpebras abertas parcialmente, sem evidência dos bulbos oculares (Figura 1A e B). Todos os parâmetros vitais avaliados estavam dentro dos limites de normalidade. O exame neurológico revelou diminuição de todas as reações posturais e proprioceptivas, além de depressão do nível de consciência. Não foram observadas alterações em nervos cranianos além do óptico. O exame ultrassonográfico do crânio confirmou anoftalmia bilateral e hidrocefalia. As imagens encefálicas exibiram atrofia e retração do parênquima encefálico, levando à diminuição evidente da espessura do manto, representado pelo tecido cerebral dorsal aos ventrículos laterais, fato também relatado por HUDSON et al. (1990). O parênquima restante apresentava ecotextura preservada, com dilatação das porções do sistema ventricular, incluindo os ventrículos laterais e terceiro ventrículo, normalmente não visualizados, de forma simétrica e bilateral (Figura 1C). As medidas do sistema ventricular estão na tabela 1 . O paciente não voltou às consultas de retorno, não sendo prescrito o tratamento e nem acompanhada a evolução do caso.
O termo anoftalmia clínica é empregado quando o bulbo ocular não é encontrado no exame clínico (SPIESS, 2007), sendo uma alteração rara em cães e gatos (MOULD, 2002; CHRISMAN et al., 2005; DEWEY, 2006; SPIESS, 2007; COOK, 2007; FERNÁNDEZ; BERNARDINI, 2010). Nesse caso, não foram encontradas evidências de estruturas oculares nos exames clínico e ultrassonográfico. A ocorrência de microftalmia (bulbo ocular anormalmente pequeno) pode estar associada à utilização de griseofulvina durante a prenhez (CHRISMAN et al., 2005), contudo, no presente relato, não houve a utilização do antifúngico.

A anoftalmia verdadeira resulta da falha no desenvolvimento do encéfalo primitivo, em estágio anterior ao da formação dos sulcos ópticos (SLATTER, 2005), provocada por defeito na formação da vesícula óptica primária (MOULD, 2002; SPIESS, 2007). O animal do presente relato encontrava-se em bom estado geral, porém, segundo SLATTER (2005), esse tipo de malformação geralmente leva ao nascimento de um filhote não viável.

A ultrassonografia encefálica (através de fontanelas abertas, dorsal ou média ou defeitos da calvária) e imagens obtidas por tomografia computadorizada e ressonância magnética têm suplantado amplamente os métodos mais invasivos de diagnóstico de ventriculomegalia, como

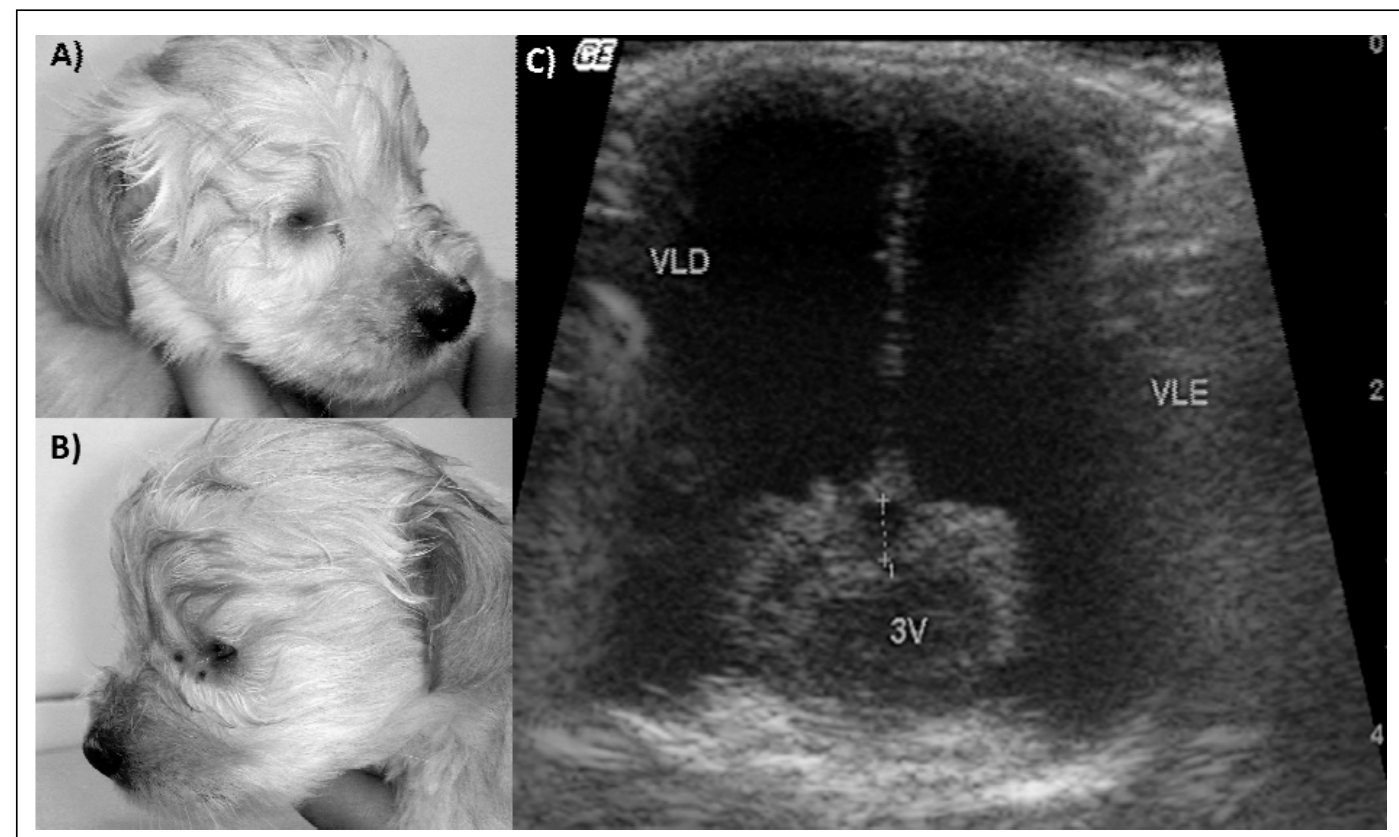

Figura 1 - Pálpebras abertas parcialmente, sem evidência dos bulbos oculares, (A): lado direito, (B): lado esquerdo, (C): imagem ultrassonográfica do crânio evidenciando dilatação de ventrículos laterais direito (VLD), esquerdo (VLE) e terceiro ventrículo (3V). 
Anoftalmia clínica bilateral associada à hidrocefalia congênita em cão.

Tabela 1 - Valores do sistema ventricular mensurados no exame ultrassonográfico.

\begin{tabular}{lcc}
\hline Estrutura & Mensuração $(\mathrm{cm})$ & Valores de referência* \\
\hline Manto encefálico & 0,34 & $1,63-2,06$ \\
Ventrículo lateral direito & 2,17 & máx 0,35 \\
Ventrículo lateral esquerdo & 2,15 & máx 0,35 \\
Terceiro ventrículo & 0,43 & Normal não mensurável \\
Proporção ventrículo/manta & 6,38 & máx 0,14 \\
Proporção ventrículo/hemisfério & 0,62 & máx 0,19 \\
\hline
\end{tabular}

*(SPAULDING \& SHARP, 1990).

ventriculografia contrastada (STADES et al., 1999). Neste caso, a ultrassonografia encefálica confirmou o diagnóstico de hidrocefalia e de anoftalmia.

O cão deste estudo apresentava fontanela persistente e não se evidenciou estrabismo, devido à anoftalmia. Cães com fontanela persistente e calvária fina são mais suscetíveis à hemorragia decorrente de traumatismos cranianos (GRAHN \& PEIFFER, 2007), porém, não foi possível a realização de tratamento e nem a observação da evolução do quadro clínico do animal.

Este é o primeiro relato da associação de hidrocefalia com anoftalmia clínica, em cão, no Brasil. A realização do exame neonatal e o conhecimento das malformações neurológicas e oftálmicas são essenciais para a detecção precoce das anomalias em cães.

\section{COMITÊ DE ÉTICA E BIOSSEGURANÇA}

O número do protocolo do comitê de ética é: 198/2010

\section{REFERÊNCIAS}

CHRISMAN, C. et al. Demência, estupor e coma. In: Neurologia para o clínico de pequenos animais. São Paulo: Roca, 2005. p.41-82.

COOK, C.S. Ocular embryology and congenital malformations. In: GELATT, K.N. Veterinary ophthalmology. 4.ed. Ames: Blackwell, 2007. p.3-36.

DEWEY, C.W. Encefalopatias: distúrbios cerebrais. In: Neurologia de cães e gatos: guia prático. São Paulo: Roca, 2006. p.59-99.

FERNÁNDEZ, V.L.; BERNARDINI, M. Anomalias congênitas. In: _ Neurologia de cães e gatos. São Paulo: MedVet, 2010. p.227-260.

FILGUEIRAS, R.R. et al. Long-term evaluation of a new ventriculoperitoneal shunt valve system in a dog. Journal of Veterinary Emergency and Critical Care, v.19, v.6, p.623628, 2009. Disponível em: <http://www.ncbi.nlm.nih.gov/ pubmed/20017769>. Acesso em: 14 nov. 2010. doi:10.1111/ j.1476-4431.2009.00484.x.

GELATT, K.N. Doenças e cirurgia da órbita do cão. In: Manual de oftalmologia veterinária. Barueri: Manole, 2003. p.27-46.

GRAHN, B.H.; PEIFFER, R.L. Fundamentals of veterinary ophthalmic pathology. In: GELATT, K.N. Veterinary ophthalmology. 4.ed. Ames: Blackwell, 2007. p.355-437.

HUDSON, J.A. et al. Neurosonography. Veterinary Clinics of North America: Small Animal Practice, v.28, n.4, p.943958, 1998. Disponível em: <http://www.ncbi.nlm.nih.gov/ pubmed/969862>1. Acesso em: 14 nov. 2010.

MOULD, J.R.B. The orbit and globe. In: Manual of small animal ophthalmology. 2.ed. Gloucester: BSAVA, 2002. p.60-77.

NARFSTROM, K. et al. Visual impairment. In: PEIFFER, Jr, R.L.; PETERSEN-JONES, S.M.P. Small animal ophthalmology: a problem-oriented approach. 3.ed. London: Saunders, 2001. p.103-176.

PRIESTER, W.A. Congenital ocular defects in cattle, horses, cats, and dogs. Journal of American Veterinary Medical Association, v.160, n.11, p.1504-1511, 1972. Disponível em: <http://www.ncbi.nlm.nih.gov/pubmed/4623843>. Acesso em: 22 dez. 2010.

SLATTER, D. Desenvolvimento e anormalidades congênitas. In: ______. Fundamentos de oftalmologia veterinária. 3.ed. São Paulo: Roca, 2005. p.23-26.

SPAULDING, K.A.; SHARP, N.J.H. Ultrasonographic imaging of the lateral cerebral ventricles in the dog. Veterinary Radiology and Ultrasound, v.31, n.2, p.59-64, 1990. Disponível em: <http://onlinelibrary.wiley.com/doi/10.1111/ j.17408261.1990.tb00783.x/abstract>. Acesso em: 08 nov. 2010. doi: 10.1111/j.1740-8261.1990.tb00783.x.

SPIESS, B.M. Diseases and surgery of the canine orbit. In: GELLAT, K.N. Veterinary ophthalmology. 4.ed. Ames: Blackwell, 2007. p.539-562.

STADES, F.C. et al. Globo. In: Fundamentos de oftalmologia veterinária. São Paulo: Manole, 1999. p.104106. 